\title{
Direction of Arrival Estimation Method in Underdetermined Condition
}

\author{
Zhang Youzhi ${ }^{a}$, Li Weibo ${ }^{b}$, Wang Hanli ${ }^{c}$ \\ Naval Aeronautical Engineering Institute, Yantai, China, 264000 \\ awebblee2009@sina.com, byrerzyz@163.com, gyoei@qq.com
}

Keywords: W-disjoint orthogonality; Underdetermined; Direction of arrival estimation

\begin{abstract}
Because some signals have W-disjoint orthogonality, this paper proposes a signal direction of arrival estimation method in underdetermined conditions. This method is mainly based on the principle that signals of different directions lead to different time delay between the sensors, construct a potential function by receiving signal phase difference, and local maximum value location of the potential function corresponds to the signal direction of arrival. The simulation results show that the method can still obtain better estimation results in the case of only using two sensors.
\end{abstract}

\section{Introduction}

Direction of arrival (DOA) estimation is one of the important contents of array signal processing and it has been widely used in radar, sonar, communication, seismic exploration, radio astronomy, and other fields. Main approach to estimate the direction of arrival is beam-forming technology. But for beam-forming, because of the limitation of Rayleigh limit, after array length is determined, its resolution is sure [1]. Traditional beam-forming often requires more sensors to discern the two close goals in the direction. Although some high resolution algorithms such as MUSIC and ESPRIT can break through the limitation of Rayleigh limit, transmission channel in array signal processing is completely unknown and time-varying, which limits the use of high-resolution direction estimation in actual environment [2]

In recent years, the direction of arrival blind estimation method development enables direction of arrival estimation under the condition of unknown channel characteristics, which has good application prospect. But under underdetermined conditions, the method does not work.

The so-called "underdetermined" namely is that the number of sensors is less than source signals. Under underdetermined condition, certain features of the source signals must be used to realize mixing matrix estimation and source signal separation, such as sparsity or W-disjoint orthogonality, etc. Scott Richard and others put forward DUET (Degenerate Unmixing and Estimation Technique) method [3] for part signal's W-disjoint orthogonality, which can use two sensors to achieve any number of mixed signals' separation. On the basis of the method, this paper proposes a new signal direction of arrival estimation method, which can make use of two sensors to achieve multiple sources' signal direction of arrival estimation.

\section{Signal mixed models and source hypothesis}

\section{Signal mixed model.}

If not considering the influence of signal's amplitude attenuation, multiplicity and noise between sensors, sensor signals will be the sum of the source signals with different time delay.

For two adjacent sensors, the mixed model is:

$$
\begin{aligned}
& x_{1}(t)=s_{1}(t)+\cdots+s_{n}(t) \\
& x_{2}(t)=s_{1}\left(t-\delta_{1}\right)+\cdots+s_{n}\left(t-\delta_{n}\right)
\end{aligned}
$$

In it, $\delta_{j}$ is the time delay of source signal $s_{j}(t)$ between the two sensors. Set source signal $s_{j}(t)$ 's direction of $\operatorname{arrival} \theta_{j}$, then signal delay $\delta_{j}$ shall satisfy equation (2). 


$$
\delta_{j}=\Delta \cdot \sin \theta_{j} / C
$$

In it, $\Delta$ is the spacing between two sensors and $c$ is signal transmission speed. After get the source signal's time delay estimation, we can get the signal's direction of arrival estimation.

Source hypothesis.

Assume that the source signal satisfies the following conditions:

1) Source signal belongs to narrowband signal.

2) Source signal's short-time Fourier transformation pair wise orthogonal, namely there is Wdisjoint orthogonality between source signals.

Give a window function $W(t)$, $\operatorname{signal}_{j}(t)$ 's short-time Fourier transform (STFT) [4] is:

$$
F^{W}\left(s_{j}(\cdot)\right)(\omega, \tau)=\int_{-\infty}^{\infty} W(t-\tau) s_{j}(t) e^{-j \omega t} d t
$$

In Literature [4] the short time Fourier transforms is called W decomposition. It is simply written as $S_{j}^{W}(\omega, \tau)$ in this paper. Narrow-band signal's time delay is much smaller than the bandwidth reciprocal. Its effect is equivalent to that baseband signal produces a phase shift, and its short-time Fourier transform should satisfy the following equation [5]:

$$
F^{W}\left(s_{j}(\cdot-\delta)\right)(\omega, \tau)=e^{-j \omega \delta} \cdot F^{W}\left(s_{j}(\cdot)\right)(\omega, \tau)
$$

If signal $s_{1}(t), s_{2}(t)$ 's short-time Fourier transform is orthogonal, we call $s_{1}(t), s_{2}(t)$ W-disjoint orthogonal. The intuitive sense of so-called "W-disjoint orthogonality" is that any point in timefrequency plane only has up to one signal which is effective, and other signals' influence can be neglected. Its mathematical expression is:

$$
S_{1}^{W}(\omega, \tau) \cdot S_{2}^{W}(\omega, \tau)=0, \forall \omega, \tau
$$

\section{Direction of arrival estimation}

If source signal strictly meets W-disjoint orthogonal, signals received at any one point in time and frequency domain at the maximum is a mixture of source signals. That is, for any $(\omega, \tau)$, if $X_{1}^{W}(\omega, \tau) \neq 0$, there is a source signal $s_{j}$ which meets:

$$
\left\{\begin{array}{l}
S_{j}^{W}(\omega, \tau) \neq 0, \\
S_{k}^{W}(\omega, \tau)=0,
\end{array}(k=1, \cdots, n ; \quad k \neq j)\right.
$$

At this point,

$$
\begin{aligned}
{\left[\begin{array}{l}
X_{1}^{W}(\omega, \tau) \\
X_{2}^{W}(\omega, \tau)
\end{array}\right] } & =\left[\begin{array}{ccc}
1 & \cdots & 1 \\
e^{-j \omega \delta_{1}} & \cdots & e^{-j \omega \delta_{n}}
\end{array}\right] \cdot\left[\begin{array}{c}
S_{1}^{W}(\omega, \tau) \\
\vdots \\
S_{n}^{W}(\omega, \tau)
\end{array}\right] \\
& =\left[\begin{array}{c}
1 \\
e^{-j \omega \delta_{j}}
\end{array}\right] \cdot S_{j}^{W}(\omega, \tau)
\end{aligned}
$$

As a result,

$$
\frac{X_{1}^{W}(\omega, \tau)}{X_{2}^{W}(\omega, \tau)}=e^{j \omega \delta_{j}}
$$


This makes the plural phase $\frac{X_{1}^{W}(\omega, \tau)}{X_{2}^{W}(\omega, \tau)}$, s angle $\hat{\theta}(\omega, \tau)$. If the distance between two sensors is less than half wavelength of the signal, its delay $\delta_{j}$ should satisfy equation (8).

$$
\delta_{j}=\frac{1}{\omega} \cdot \hat{\theta}(\omega, \tau)=\frac{1}{\omega} \angle \frac{X_{1}^{W}(\omega, \tau)}{X_{2}^{W}(\omega, \tau)}
$$

So, $\forall(\omega, \tau)$. If $X_{1}^{W}(\omega, \tau) \neq 0$, then

$$
\frac{1}{\omega} \cdot \hat{\theta}(\omega, \tau) \in\left\{\delta_{1}, \ldots, \delta_{n}\right\}
$$

So if source signals strictly meet W-disjoint orthogonality, classify $1 / \omega \cdot \hat{\theta}(\omega, \tau)$ and get any time delay parameter estimation. And this also can complete source signal separation. But in practice, source signals can only be approximate to meet $\mathrm{W}$-disjoint orthogonal hypothesis, adding to the noise influence, $1 / \omega \cdot \hat{\theta}(\omega, \tau)$ can only be near time delay parameters according to probability distribution.

In order to complete source signals' direction of arrival estimation, this paper adopts Literature [6]'s potential function method.

$$
\Phi(\theta)=\sum_{\omega, \tau} l(\omega, \tau) \phi(\theta-\hat{\theta}(\omega, \tau))
$$

In it, there is $\phi(\theta-\hat{\theta}(\omega, \tau))=\exp \left(-\frac{\sin ^{2}(\theta-\hat{\theta}(\omega, \tau))}{2 \sigma^{2}}\right)$ and $l(\omega, \tau)=X_{1}^{W}(\omega, \tau)^{2}+X_{2}^{W}(\omega, \tau)^{2}$.

The location's local maximum value of potential function is corresponding to each source signal's direction of arrival.

\section{Simulation experiment and result analysis}

\section{Experiment 1}

In order to test the algorithm effectiveness, analyze algorithm performance in simulation underwater acoustic environment. Choose three single frequency signals as source signals whose frequencies are $3 \mathrm{k}, 3.5 \mathrm{k}$ and $4 \mathrm{k}$. Signal sources' angle difference between two is identical, in which the middle signal source is located right in front of the sensor array. Set array elements spacing to $5 \mathrm{~cm}$, sound velocity in water is set to $1430 \mathrm{~m} / \mathrm{sec}$, sampling frequency $16 \mathrm{KHZ}$ and data length 160000. Window function uses Hamming window with 1024 points, there are 512 points overlap each other between adjacent two window functions. Noises are respectively $-5 \mathrm{db}, 0 \mathrm{db}, 5$ $\mathrm{db}, 10 \mathrm{db}, 15 \mathrm{db}$, and $20 \mathrm{db}$ additive white Gaussian noise. Do 50 times simulation test for each case, and the algorithm's recognition rate in each case and the average error are shown in Table 1.

From the experiment results, we can see that when the angle difference of source signal is greater than 3 degrees, the average error of this method's direction of arrival estimation under the condition that signal-to-noise ratio is higher than $15 \mathrm{db}$ is 0.09 degrees (The error is mainly due to potential function discretization), and the recognition rate is $100 \%$. And the Angle difference between source signal certain circumstances, with the loss of the signal to noise ratio, the recognition rate of the proposed algorithm is reduced, error is increased. When SNR is reduced to a certain degree, the algorithm can't distinguish the direction of the three sources, and only produce a crest near 0 degree.

It can be seen from Table 1, noise is the main factor affecting the algorithm, and the algorithm can obtain higher precision estimation under certain signal to noise ratio. 
Table 1 Angle estimation's average error and recognition rate under various circumstances

\begin{tabular}{|c|c|c|c|c|c|c|c|}
\hline $\begin{array}{c}\text { Angle } \\
\text { difference }\end{array}$ & $\begin{array}{l}\text { Noise } \\
\text { intensity }\end{array}$ & $-5 \mathrm{db}$ & $0 \mathrm{db}$ & $5 \mathrm{db}$ & $10 \mathrm{db}$ & $15 \mathrm{db}$ & $20 \mathrm{db}$ \\
\hline \multirow{2}{*}{$3^{\circ}$} & $\begin{array}{c}\text { Average } \\
\text { error }\end{array}$ & & & $1.23^{\circ}$ & $0.47^{\circ}$ & $0.09^{\circ}$ & $0.09^{\circ}$ \\
\hline & $\begin{array}{l}\text { Recognition } \\
\text { rate }\end{array}$ & & & $40 \%$ & $80 \%$ & $100 \%$ & $100 \%$ \\
\hline \multirow{2}{*}{$5^{\circ}$} & $\begin{array}{l}\text { Average } \\
\text { error }\end{array}$ & & $2.31^{\circ}$ & $0.89^{\circ}$ & $0.24^{\circ}$ & $0.09^{\circ}$ & $0.09^{\circ}$ \\
\hline & $\begin{array}{l}\text { Recognition } \\
\text { rate }\end{array}$ & & $22 \%$ & $56 \%$ & $92 \%$ & $100 \%$ & $100 \%$ \\
\hline \multirow{2}{*}{$7^{\circ}$} & $\begin{array}{l}\text { Average } \\
\text { error }\end{array}$ & & $1.21^{\circ}$ & $0.78^{\circ}$ & $0.12^{\circ}$ & $0.09^{\circ}$ & $0.09^{\circ}$ \\
\hline & $\begin{array}{l}\text { Recognition } \\
\text { rate }\end{array}$ & & $42 \%$ & $64 \%$ & $100 \%$ & $100 \%$ & $100 \%$ \\
\hline \multirow{2}{*}{$9^{\circ}$} & $\begin{array}{c}\text { Average } \\
\text { error }\end{array}$ & & $1.2^{\circ}$ & $0.23^{\circ}$ & $0.09^{\circ}$ & $0.09^{\circ}$ & $0.09^{\circ}$ \\
\hline & $\begin{array}{l}\text { Recognition } \\
\text { rate }\end{array}$ & & $52 \%$ & $94 \%$ & $100 \%$ & $100 \%$ & $100 \%$ \\
\hline \multirow{2}{*}{$11^{\circ}$} & $\begin{array}{c}\text { Average } \\
\text { error }\end{array}$ & $3.1^{\circ}$ & $1.5^{\circ}$ & $0.18^{\circ}$ & $0.09^{\circ}$ & $0.09^{\circ}$ & $0.09^{\circ}$ \\
\hline & $\begin{array}{l}\text { Recognition } \\
\text { rate }\end{array}$ & $12 \%$ & $56 \%$ & $100 \%$ & $100 \%$ & $100 \%$ & $100 \%$ \\
\hline
\end{tabular}

Table 2 Array element number required to distinguish three signal source directions under the condition when noise is $10 \mathrm{db}$

\begin{tabular}{|c||c|c|c|c|c|c|}
\hline \hline \multirow{2}{*}{$\begin{array}{c}\text { Angle } \\
\text { difference }\end{array}$} & \multicolumn{2}{|c|}{ Conventional method } & \multicolumn{2}{|c|}{ Self-adaptive method } & \multicolumn{2}{|c|}{$\begin{array}{c}\text { Array space high } \\
\text { resolution method }\end{array}$} \\
\cline { 2 - 7 } & Capon & Batlette & LMS & LCMV & MUSIC & ESPRIT \\
\hline $3^{\circ}$ & 35 & 35 & 31 & 18 & 12 & 8 \\
\hline $5^{\circ}$ & 21 & 21 & 19 & 12 & 8 & 5 \\
\hline $7^{\circ}$ & 15 & 15 & 14 & 10 & 5 & 4 \\
\hline $9^{\circ}$ & 12 & 12 & 11 & 9 & 5 & 4 \\
\hline
\end{tabular}

\section{Experiment 2}

Calculate array element number required to estimate three source signal direction of arrival under the condition of different angle difference by all kinds of direction of arrival estimation 
methods. Set signal-to-noise ratio as $10 \mathrm{db}$, other parameters settings are the same of experimental one. Under the condition of different angle difference, distinguish three source signal direction of arrival's required minimum array element number, which is shown in Table 2.

It can be seen from Table 2 that at present the most commonly used method of direction of arrival estimation need more array element number to distinguish the direction of the three goals. And in the same condition, the text method only needs two array elements.
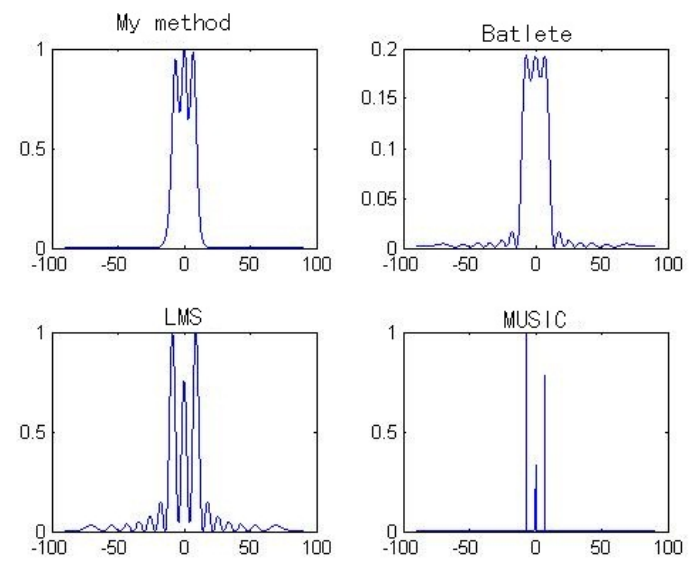

Figure 1 Direction estimate figure of all direction of arrival estimation method

Figure 1 shows direction estimate figure of all direction of arrival estimation when signal source angle difference is $7^{\circ}$ and array element number is 16. The method only uses two sensors' array elements. It can be seen from the diagram that although this method only uses two arrays elements data, it can still get a good estimation result, especially relative to conventional beam forming.

\section{Conclusion}

Some signals have W-disjoint orthogonality. Based on DUET underdetermined blind source separation method, a new method of signal direction of arrival estimation is proposed. The method estimates direction of arrival of signal source by potential function constructed by phase difference receiving signals, which can break through the limitation of conventional beam forming's Rayleigh limit, and only two sensors array elements are needed to complete multiple source signal direction of arrival estimation. Simulation results show that under certain condition, the signal-to-noise ratio of this method can get better precision estimation.

\section{References}

[1] Zhang Xianda, Bao Zheng. Communication signal processing [M]. Beijing: National Defence Industry Press, 2000,12, 326.

[2] Jia Yongkang. Array signal processing robustness research. Ph.D. Dissertation, Xi 'an University of Electronic Science and Technology, 1996, 5.

[3] A. Jourjine, S. Rickard, and Ö. Y1lmaz, "Blind separation of W-disjoint orthogonal signals: Demixing N sources from 2 mixtures," in Proc. IEEE Int. Conf. Acoust., Speech, Signal Process., vol. 5, Istanbul, Turkey, June 5-9, 2000, pp. 2985-2988.

[4] I. Daubechies, "ten Lectures on Wavelets" Philadelphia, PA: SIAM, 1992.

[5]R. Balan, J. Rosca, S. Rickard, and J. O'Ruanaidh, "The influence of windowing on time delay estimates," in Proceedings of the 35th Annual Conference on Information Sciences and Systems (CISS2000), Princeton, NJ, March 15-17 2000, vol. 1,pp. WP1-(15-17).

[6] P. Bofill, M. Zibulevsky, "Underdetermined blind source separation using sparse representations," in signal processing 81(2001), 2353-2362. 